\title{
Behavioral and quantitative mitochondrial proteome analyses of the effects of simvastatin: implications for models of neural degeneration
}

\author{
Ilse S. Pienaar - Timothy Schallert • \\ Suzél Hattingh · William M. U. Daniels
}

Published online: 21 August 2009

(C) Springer-Verlag 2009

\section{Erratum to: J Neural Transm (2009) 116:791-806 DOI 10.1007/s00702-009-0247-4}

The correct abstract is given here:

\begin{abstract}
The 3-hydroxy-3-methylglutaryl coenzyme A reductase inhibitor, simvastatin, is used for lowering elevated low-density lipoprotein cholesterol concentrations. This translates into reduced cardiovascular disease-related morbidity and mortality, while the drugs' anti-oxidant and anti-inflammatory properties have earmarked it as a potential treatment strategy against various neurological conditions. Statins have been shown to protect neurons from degeneration in a number of animal models. Although no mechanism completely explains the multiple benefits exerted by statins, emerging evidence suggests that in some
\end{abstract}

The online version of the original article can be found under doi:10.1007/s00702-009-0247-4.

I. S. Pienaar $(\square)$

MRC Functional Genomics Unit, Department of Physiology, Anatomy and Genetics, University of Oxford, South Parks Road, Oxford OX1 3QX, UK

e-mail: Ilse.pienaar@dpag.ox.ac.uk

I. S. Pienaar · S. Hattingh

Department of Medical Physiology, University of Stellenbosch,

Tygerberg, South Africa

T. Schallert

Departments of Psychology and Neurobiology,

University of Texas at Austin, Austin, USA

W. M. U. Daniels

Department of Medicine, University of Kwazulu-Natal,

Congella, South Africa degenerative and brain injury models, mitochondrial impairment may play a contributive role. However, evidence lacks to support a directly influencing role for statins on mitochondria-related proteins and motor behavior. Mitochondrial dysfunction may increase oxygen free radical production, which in turn leaves cells susceptible to energy failure, apoptosis and related events which could prove fatal. The potential link between simvastatin treatment and mitochondrial function would be supported if key mitochondrial proteins were altered by simvastatin exposure. Using mass spectroscopy (MS), we identified 24 mitochondrial proteins that differed significantly $(P<0.05)$ in relative abundancy as a result of simvastatin treatment. The identified proteins represented many facets of mitochondrial integrity, with the majority forming part of the electron transport chain machinery, which is necessary for energy production. In a follow-up study, we then addressed whether simvastatin is capable of altering sensorimotor function in a mitochondrial toxin-induced animal model. Rats were pre-treated with simvastatin for 14 days, followed by a single unihemispheric (substantia nigra; $\mathrm{SN}$ ) injection of rotenone, a mitochondrial complex I (Co-I) inhibitor. Results showed that simvastatin improved motor performance in rotenone-infused rats. The data are consistent with the possibility that alteration of mitochondrial function may contribute to the beneficial effects associated with statin use. 